\title{
Hypoxic Training in Obese Mice Improves Metabolic Disorder
}

\author{
Ru Wang ${ }^{1 *}$, Shanshan Guo ${ }^{1 \dagger}$, Haili Tian ${ }^{1+}$, Yiru Huang ${ }^{2,3}$, Qin Yang ${ }^{1}$, Kewei Zhao ${ }^{1}$, \\ Chia-Hua Kuo ${ }^{4}$, Shangyu Hong ${ }^{2}$, Peijie Chen ${ }^{1 *}$ and Tiemin Liu ${ }^{1,2,3 *}$
}

${ }^{1}$ School of Kinesiology, Shanghai University of Sport, Shanghai, China, ${ }^{2}$ State Key Laboratory of Genetic Engineering, Department of Endocrinology and Metabolism, School of Life Sciences, Zhongshan Hospital, Fudan University, Shanghai, China, ${ }^{3}$ State Key Laboratory of Pharmaceutical Biotechnology, Nanjing University, Nanjing, China, ${ }^{4}$ Laboratory of Exercise Biochemistry, University of Taipei, Taipei, Taiwan

Hypoxic training has been reported to lower obesity morbidity without clear underlying mechanisms. This study investigates the effect of hypoxic training on metabolic changes, particularly, on liver metabolism of high fat diet (HFD)-induced obese mice. We compared the hypoxic training group with normoxic sedentary, normoxic training, and hypoxic sedentary groups. Body weight, fat mass, glucose tolerance and liver physiology were determined after 4 weeks intervention. In both normoxic training and hypoxic training groups, body weight was lower than the normoxic sedentary group, with less fat mass. Insulin sensitivity was improved after hypoxic training. Moreover, liver metabolomics revealed insights into the protective effect of hypoxic training on HFD-induced fatty liver. Taken together, these findings provide a molecular metabolic mechanism for hypoxic training.

Keywords: hypoxic training, obesity, metabolomics, liver, metabolism

\section{INTRODUCTION}

The recent observation that in obese subjects adipose tissue becomes hypoxic and triggers inflammation and obesity-associated diseases (1) has generated inquiries as to the potential of oxygen therapy as a tool for weight management $(2,3)$. At the same time, there is research that indicates that hypobaric hypoxia and normobaric hypoxia can lead to weight loss and lower the risk of metabolic syndrome, respectively (4-6). While the mechanism underlying these observations is still unknown, combining hypoxia and exercise training might provide a cost-effective strategy for reducing body weight and improving metabolic health in obese humans.

Previously, hypoxic training was used to increase exercise performance of athletes. "Live high-train low" (normobaric hypoxia living and normoxic training) and "live low-train high" (normoxic living and hypobaric hypoxia training) were the most popular methods (7). It has been established that, long-term severe hypoxia exposure causes impairment of skeletal muscle and vascular endothelial function, and vascular hemodynamics $(8,9)$. At the tissue level, it was reported that acclimatization to hypoxia (10 days at $5,500 \mathrm{~m}$ ) reduced the aerobic capacity of the rat skeletal muscle (gastrocnemius) through a fall in citrate synthase activity (10). Another study has shown that normobaric hypoxic $\left(\mathrm{FIO}_{2}=15 \%\right)$ training enhanced the activation of satellite cells and angiogenesis of the thoroughbred horse skeletal muscle (9).

In obese subjects, the liver-an organ, critical to glycogenesis, glycogen storage, lipogenesis, fatty acid oxidation, lipolysis and decomposition of erythrocytes- is in a "hypermetabolic state." Especially in obese subjects, endogenous metabolites are altered in response to nutrition 
consumption and energy expenditure. Therefore, metabolomics provides a potential platform to monitor changes in liver metabolites under hypoxic training. A metabolomics approach, to characterize variations in metabolite profile and identify biomarkers level with/without hypoxic training on obese subjects, would contribute to the dearth of literature.

To date, the combination of hypoxia and exercise has mainly been investigated in normal weight (BMI $<25 \mathrm{~kg} / \mathrm{m}^{2}$ ) or lean individuals. Very few studies have included obese subjects-those that have not focused on metabolic or body composition changes associated with hypoxic exercise. Specifically, in recent studies that included obese subjects, compared to non-obese subjects, the obese group reported greater reductions in serum reactive oxygen species (ROS) after oxidative training $(11,12)$.

The purpose of this study is to investigate the metabolic effects of hypoxic training on high fat diet (HFD)-induced obese mice. We hypothesize that, compared to normobaric hypoxia and normoxic training, hypoxic training would result in greater weight loss and changes in liver glucose and lipid metabolism in obese mice.

\section{MATERIALS AND METHODS}

\section{Animal Subjects}

Four-weeks-old healthy male C57BL/6J mice were obtained from the Experimental Animal Center of Shanghai Second Military Medical University. All mice were housed under standard laboratory conditions ( $12 \mathrm{~h}$ on/off; lights on at 8:30 a.m.) and a temperature-controlled environment $\left(22-24^{\circ} \mathrm{C}\right)$ with food and water available ad libitum in the SPF animal research center of Shanghai University of Sports (SYXK 2014-0002). All experiments were performed in accordance with the guidelines, established by Science Research Ethics Committee at the Shanghai University of Sports (No. 2015013) and approved by the Animal Care and Use Committee at the Shanghai University of Sports. Mice were fed with a HFD (Research Diet, \#D12492; $60 \% \mathrm{kcal}$ from fat, $5.24 \mathrm{kcal} / \mathrm{g}$ ) beginning at 5 weeks old.

\section{Animal Model Preparation}

After 13 weeks on HFD, the 18-weeks-old mice were randomly divided into four treatment groups: normoxic sedentary (S), normoxic training (NT), hypoxic sedentary $(\mathrm{H})$, and hypoxic training $(\mathrm{HT})$. While the mice were divided into four treatment groups, they were weight-matched. Treatments lasted for 4 weeks.

\section{Training and Hypoxic Intervention Treadmill Training Protocol}

Treadmill training was performed as previously described with modifications (13). Briefly, at 18 weeks of age, mice were acclimated to treadmill training for 3 days. From week 19 onwards, mice trained 6 days/week (Monday-Saturday) with a daily run time of $90 \mathrm{~min}$. Each run began with $8 \mathrm{~min}$ at a speed of $6 \mathrm{~m} / \mathrm{min}$. From minute 9 to minute 30 , the speed was gradually increased $(1 \mathrm{~m} / \mathrm{min}$ increase every $3 \mathrm{~min}$ ) until a maximum speed of $14 \mathrm{~m} / \mathrm{min}$ was reached at minute 30 . Mice were kept running at $14 \mathrm{~m} / \mathrm{min}$ from minute 30 to minute 90 .

\section{Establishment of Hypoxic Environment}

The TSE PhenoMaster hypoxia metabolism warehouse was used to establish a moderate and constant hypoxic experimental environment. Oxygen concentration was set at 14.7\% (although actual oxygen concentration ranged from 14.4 to $14.7 \%$ during the experiment) based on the well-established observation that altitude stress is induced at $3,000 \mathrm{~m}$ above sea level ( $\sim 14.4 \%$ oxygen concentration) and our pre-experimental data showed that humans behaved normally in food consumption and other daily activities without any adverse reaction when oxygen concentration was below $14.7 \%$. Intervention bouts were performed 8 h/day, 6 days/week (Monday-Saturday).

\section{Glucose Tolerance Tests}

After an overnight fasting, 22-weeks-old mice were treated with intraperitoneal (i.p.) injections of $2 \mathrm{~g} / \mathrm{kg}$ D-glucose. Blood glucose was measured from tail blood using a glucometer (Roche) at serial time points as indicated in figures. The areas under the curve (AUCs) were calculated using trapezoidal integration.

\section{Insulin Tolerance Tests}

After a 4-h fasting to empty the stomach, 22-weeks-old male mice received i.p. injections of insulin $(1.0 \mathrm{U} / \mathrm{kg})$. Blood glucose level was measured from tail blood as described above. The areas under the curve (AUC) were calculated using trapezoidal integration.

\section{Immunohistochemistry Oil Red O Staining}

To compare size and density of lipid droplets in mice liver, liver was stored in 4\% paraformaldehyde (Wuhan Google Biotechnology) for more than $12 \mathrm{~h}$, then embedded in OCT (Sakura), cut on a cryotome (E, Thermo) at 8-10 $\mu \mathrm{m}$ thickness and preserved in $-20^{\circ} \mathrm{C}$ freezer. Snap-frozen liver sections were fixed with $4 \%$ paraformaldehyde, washed three times in phosphate buffer saline (PBS), then incubated with oil red $\mathrm{O}$ (Wuhan Google Biotechnology) for 10-15 min, following three times washing in PBS. Sections were stained with Harris (Wuhan Google Biotechnology) right after oil red O-staining and finally washed with flowing water.

\section{Hematoxylin and Eosin Staining}

To assess general morphology of liver, liver was stored in $4 \%$ paraformaldehyde (Wuhan Google Biotechnology) for more than $12 \mathrm{~h}$, then the tissues were processed routinely for paraffin embedding, and $4-\mu \mathrm{m}$-thick sections were cut and placed on glass slides. The paraffin-embedded sections were dewaxed with xylene, washed by gradient ethanol to water, then incubated with hematoxylin and eosin (Servicebio) for $5 \mathrm{~min}$, and sealed after conventional ethanol dehydration. Finally, sections were analyzed under a Nikon light microscope at the indicated magnification.

\section{Metabolite Analysis by NMR}

\section{Preparation of Liver Samples for NMR Analysis}

Approximately $55 \mathrm{mg}$ of each sample was lysed in $600 \mu \mathrm{l}$ icecolded $80 \%$ methanol by Tissue-lyzer (QIAGEN Tissuelyzer, 
Germany) with stainless-steel beads (20 Hz for $90 \mathrm{~s}$ ) (14). The lysate was transferred to new tubes and ultraphonic for 10 times (for a duration of $60 \mathrm{~s}$ each time with a 60$\mathrm{s}$ interval between times) on ice, and the supernatant was collected by centrifugation $\left(4^{\circ} \mathrm{C}, 11,180 \mathrm{~g}, 10 \mathrm{~min}\right)$. The pellet was extracted two more times following the same procedure. Pooled supernatants were centrifuged for $10 \mathrm{~min}\left(4^{\circ} \mathrm{C}, 11,180 \mathrm{~g}\right)$ to obtain the final extracts. Before proceeding to NMR analysis, the methanol in the final extracts was removed by a rotary evaporator (SC110A, Thermo, Germany). The remaining of the evaporated extracts were lyophilized and resuspended in 550 $\mu \mathrm{l} \mathrm{Na}{ }^{+} / \mathrm{K}^{+}$buffer $\left(0.15 \mathrm{M}, 80 \% \mathrm{D}_{2} \mathrm{O}, 0.01071 \% \mathrm{TSP}, \mathrm{pH} 7.40\right)$ and cleaned by centrifugation $\left(4^{\circ} \mathrm{C}, 11,180 \mathrm{~g}, 10 \mathrm{~min}\right) ; 500 \mu \mathrm{l}$ of each sample was transferred to a 5 -mm NMR tube for ${ }^{1} \mathrm{H}$ NMR detection.

\section{NMR Spectroscopy}

NMR spectra were acquired at $298 \mathrm{~K}$ on a Bruker AVIII $600 \mathrm{MHz}$ NMR spectrometer (600.13 MHz for proton frequency) equipped with a cryogenic probe (Bruker Biospin, Germany) at $298 \mathrm{~K}$.

For liver samples we used the first increment of the NOESY pulse sequence $\left(\mathrm{RD}-90^{\circ}-\mathrm{t} 1-90^{\circ}\right.$-tm- $90^{\circ}$-acquisition; $\mathrm{t} 1=4 \mu \mathrm{s}$, $\mathrm{tm}=100 \mathrm{~ms}$ ). A total of 64 transients for each sample were collected into $32 \mathrm{~K}$ data points over a spectral width of $20 \mathrm{ppm}$ with a $90^{\circ}$ pulse length adjusted to $10.15 \mathrm{~ms}$.

\section{NMR Spectral Data Analysis}

The free induction decays were multiplied by an exponential window function with the line broadening factor of $1 \mathrm{~Hz}$ prior to Fourier transformation. Each spectrum was corrected for phase and baseline deformation manually using Topspin 2.1
A

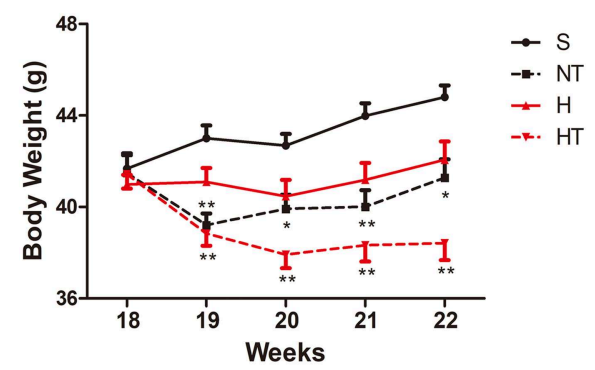

B

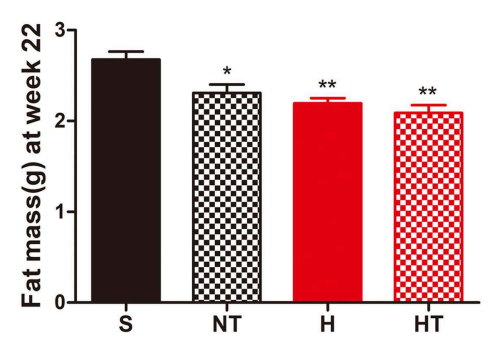

FIGURE 1 | Hypoxic training decreases body weight and fat mass. (A) Body weight curves of high-fat diet induced obese (DIO) mice on sedentary (S, $n=8)$, normoxia training (NT, $n=8)$, hypoxia $(\mathrm{H}, n=10)$, and hypoxia + training $(\mathrm{HT}, n=8)$ for 4 weeks. (B) Epididymal and perirenal fat mass in DIO mice after 4 weeks of sedentary (S, $n=8)$, normoxia training (NT, $n=7)$, hypoxia $(\mathrm{H}, n=8)$, and hypoxia + training $(H T, n=8)$ treatment at 22 weeks of age. All data are presented as mean \pm SEM. ${ }^{*} p<0.05,{ }^{* \star} p<0.01$, compared with sedentary (S) group mice.

\section{A}

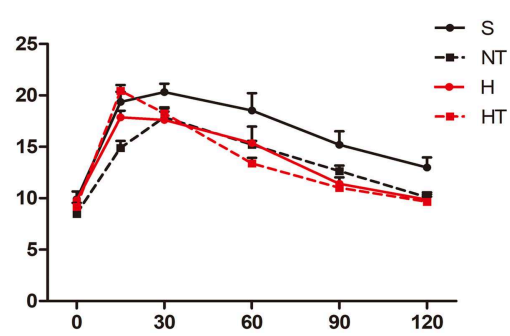

C

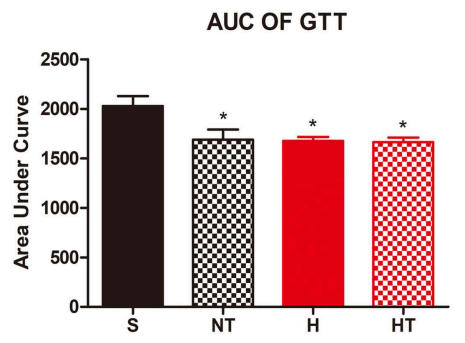

B

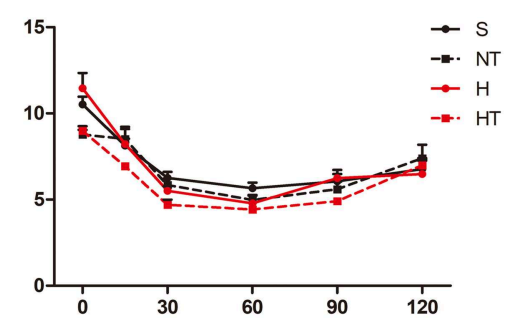

D

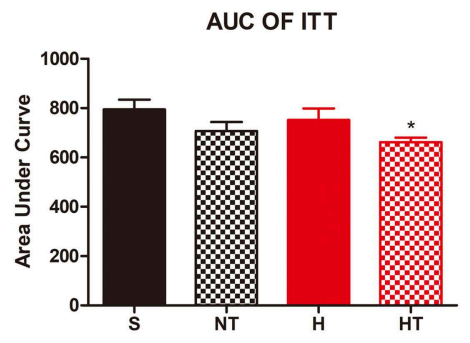

FIGURE 2 | Hypoxic training improves glucose hemostasis of HFD-fed mice. Plots for glucose tolerance tests (GTT, $2 \mathrm{~g} / \mathrm{kg} \mathrm{BW,} n=7-8$ ) in overnight fasted mice (A) and insulin tolerance tests (ITT, $1 \mathrm{U} / \mathrm{kg} \mathrm{BW}, n=7-8)$ in mice fasted for $6 \mathrm{~h}$ (B), respectively from mice in each group. (C,D) AUC values confirmed improvements of glucose tolerance in $\mathrm{HT}$ group mice and no difference of insulin tolerance in all the groups. All data are presented as Mean \pm SEM, ${ }^{*} p<0.05$, compared with sedentary (S) group mice, by two-way ANOVA. 
(Bruker Biospin) and the chemical shift (TSP at $\delta 0.00$ for liver). The spectral region $(0.5-10 \mathrm{ppm}$ for liver) was integrated into bins with a width of 0.002 ppm using AMIX package (v3.9.2, Bruker Biospin). Some unwanted signals, such as water signals ( $\delta$ 4.59-5.18 ppm) and methanol signals ( $\delta$ 3.35-3.37 $\mathrm{ppm})$, were removed (15). The range of each integration interval was $0.002 \mathrm{ppm}$. All data were normalized by the wet weight of each sample.

\section{GC-FID-MS Analysis of Fatty Acid Composition for Liver Tissue}

Liver fatty acids were methylated following the methods described previously with some modifications $(16,17)$. Briefly, $10 \mathrm{mg}$ of liver sample was mixed with the internal standard (C17:0 fatty acid methyl ester) and methyl esterification reagent for reaction. After extraction with hexane, each sample was dried and resuspended in $100 \mu \mathrm{l}$ of $\mathrm{n}$-hexane for detection. A DB-225 chromatographic column (10 m long, $0.1 \mathrm{~mm}$ internal diameter; $0.1 \mu \mathrm{m}$ coating thickness; Agilent, USA) was used, and injector port and detector temperatures were both set at $230^{\circ} \mathrm{C}$. Two groups of samples were randomly interspersed during the sampling process.

\section{Statistical Analysis}

Data are shown as mean \pm standard error of mean (SEM). An independent $t$-test was used to compare fatty acid levels between $\mathrm{S}$ and HT groups. A Kruskal-Wallis ANOVA was used for the comparison of the S, NT, H, HT groups, and the Mann-Whitney test was used for post-hoc individual group comparisons (Bonferroni correction). Statistical significance was set at $p<0.05$ for all analyses. Statistical analyses were performed in SPSS 19.0.

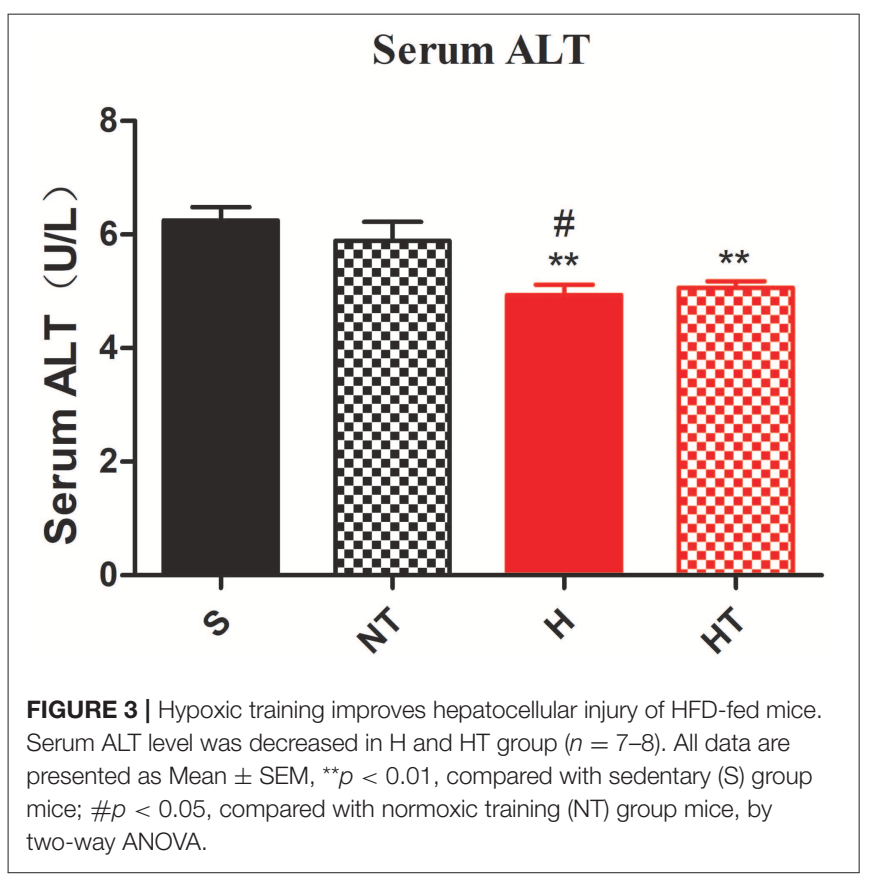

\section{RESULTS}

\section{Mice Showed Lower Body Weight After Hypoxic Training}

Following 13 weeks of a HFD, mice were randomly assigned to four treatment groups [normoxic sedentary (S), normoxic training (NT), hypoxic sedentary $(\mathrm{H})$, and hypoxic training (HT)] and body weight (BW) was measured at the end of each week during the 4 weeks treatment period (Figure 1A). Over the course of 4 weeks of treatment, hypoxic trained mice had the largest reduction of body weight followed by normoxic trained mice; hypoxic sedentary mice showed the least reductions in body weight while normoxic sedentary mice gained weight. Compared to S mice (control group), HT and NT mice showed consistent significantly lower BW at each of the measurement weeks. In contrast, $\mathrm{H}$ mice showed no significant difference in BW compared to $S$ mice and the result was consistent at each of the measurement weeks. Consistent with this, epididymal and perirenal fat mass, measured at week 22 , were also significantly lower in the three groups compared to $S$ mice (Figure 1B).

\section{Mice Have Better Glucose Homeostasis After Hypoxic Training}

We assessed the effect of hypoxic training on glucose homeostasis and found that hypoxic trained mice had improved glucose tolerance testing relative to normoxic sedentary mice (Figures 2A,C). The same result as the insulin tolerance testing, hypoxic trained mice also had improved insulin tolerance testing relative to normoxic sedentary mice (Figures 2B,D).

\section{Mice With Hypoxia Training Have Ameliorated Fatty Liver}

We also asked whether hypoxic training can ameliorate fatty liver or not. As expected, the morphology of liver from hypoxic training group was more reddish than $\mathrm{S}$ group (data not shown). Compared to $\mathrm{S}$ group, serum Alanine aminotransferase (ALT) levels in HT group were significantly decreased (Figure 3). Concomitant with this, the liver had less lipid droplets in hepatocytes in HT group compared to either S or H groups, though the lipid accumulation was similar to $\mathrm{N}$ group (Figure 4).

\section{Liver Metabolomics Reveals Differential Regulation of Lipid Metabolism by Hypoxic Training}

Our results from GC-FID-MS (Table 1) showed that, compared to $S$ group, the level of saturated fatty acid C18 in HT group mice was significantly decreased. Furthermore, HT group mice had obviously lower levels of PUFA, n3 PUFA, n6 PUFA, C20:5n3, and C22:6n3 in liver compared with $\mathrm{S}$ group. Moreover, in mice livers, hypoxic training resulted in a significant increase in the level of 3-hydroxybutytrate (Figure 5A).

\section{Hypoxic Training Affects HFD-Induced Glucose Metabolism in Liver}

NMR results indicated that hypoxic training for 4 weeks significantly increased the level of glycogen in the liver 


\section{A}

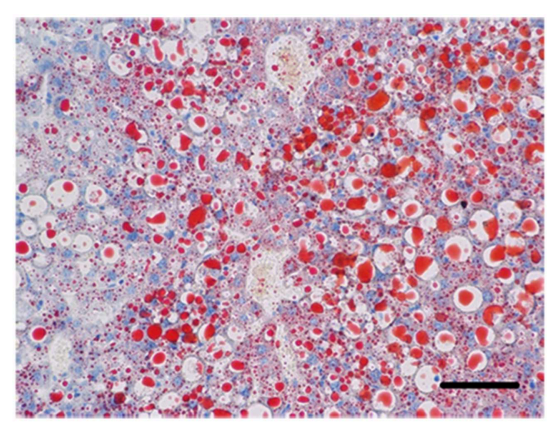

Sedentary (S)

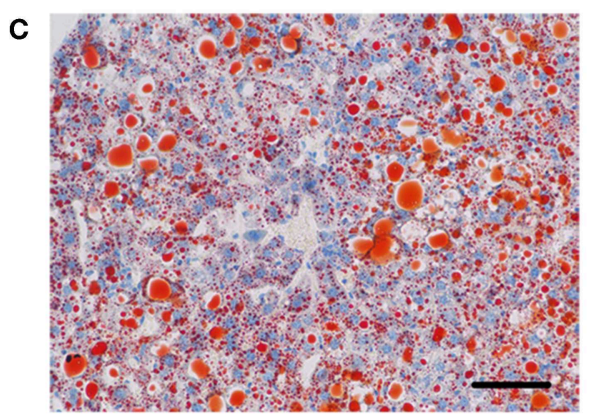

Hypoxia $(\mathrm{H})$

E

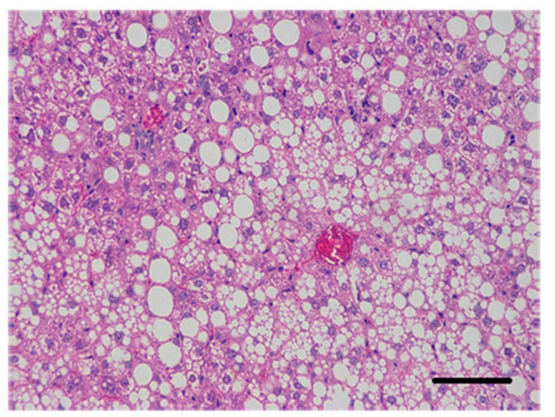

Sedentary (S)

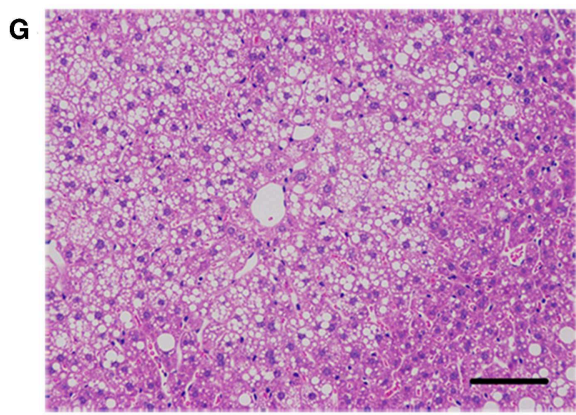

Hypoxia (H)

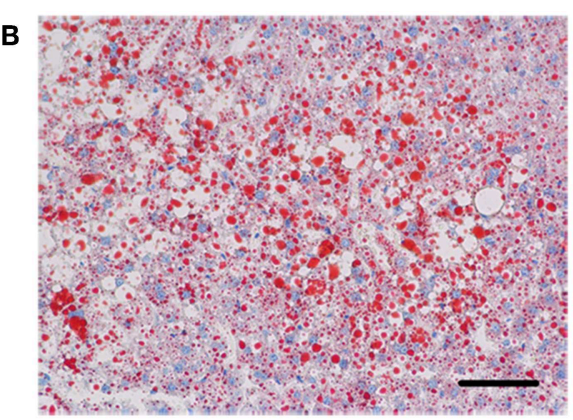

Normoxia Training (NT)

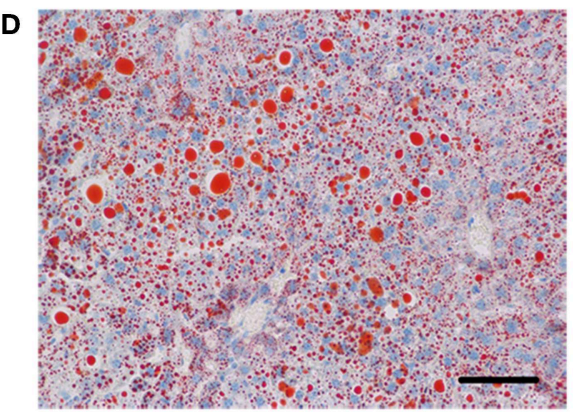

Hypoxia Training (HT)

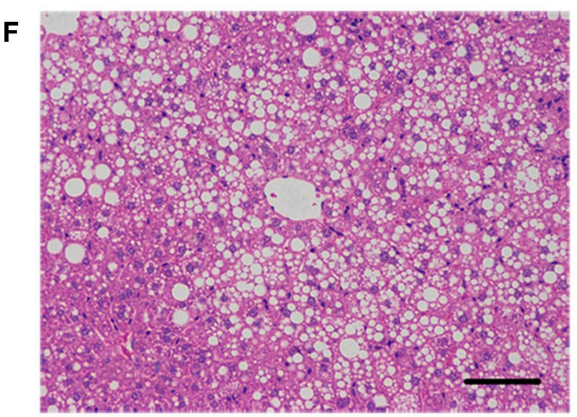

Normoxia Training (NT)

H

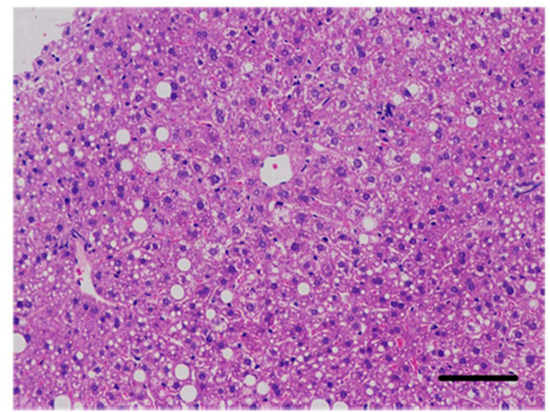

Hypoxia Training (HT)

FIGURE 4 | Effect of hypoxic training on the level of lipid droplets in mice liver sections. Representative figures of liver Oil Red O Staining from respectively mice in each group (A-D). Representative figures of liver hematoxylin-eosin staining from respectively mice in each group (E-H). Scale bar: 50 um.

of HFD-induced obese mice (Figure 5A). Additionally, hypoxic training significantly increased the levels of branched-chain amino acids including isoleucine, valine, and leucine in mouse livers. However, no significant change was found in TCA cycle between HT and S groups. Taken together, our data reveal that hypoxic training promotes liver glucose metabolism in HFD-induced obese mice (Figure 5B). 
TABLE 1 | GC-FID-MS data for fatty acids detected in mice liver.

\begin{tabular}{|c|c|c|c|}
\hline Fatty acid & S (umol/g) & HT (umol/g) & $p$-value \\
\hline C16:0 & $498.37 \pm 51.25$ & $415.27 \pm 52.82$ & 0.278 \\
\hline C16:1n7 & $43.23 \pm 5.37$ & $39.95 \pm 5.37$ & 0.673 \\
\hline C18:0 & $96.68 \pm 5.05$ & $82.04 \pm 4.33$ & $0.0495^{\star}$ \\
\hline C18:1n9 & $684.54 \pm 71.47$ & $561.83 \pm 79.33$ & 0.270 \\
\hline C18:2n6 & $245.35 \pm 14.22$ & $215.53 \pm 22.68$ & 0.284 \\
\hline C20:1n9 & $11.81 \pm 1.91$ & $8.64 \pm 1.41$ & 0.202 \\
\hline C20:3n6 & $15.73 \pm 1.33$ & $12.36 \pm 1.56$ & 0.124 \\
\hline C20:4n6 & $101.25 \pm 3.72$ & $95.25 \pm 1.99$ & 0.197 \\
\hline C20:5n3 & $1.99 \pm 0.17$ & $1.39 \pm 0.08$ & $0.009^{\star \star}$ \\
\hline C22:6n3 & $59.80 \pm 2.02$ & $51.87 \pm 0.89$ & $0.005^{\star \star}$ \\
\hline C24:0 & $1.61 \pm 0.08$ & $1.59 \pm 0.02$ & 0.850 \\
\hline $\mathrm{C} 24: 1$ & $0.70 \pm 0.12$ & $0.49 \pm 0.04$ & 0.137 \\
\hline ToFA & $1,761.05 \pm 149.70$ & $1,355.80 \pm 117.00$ & 0.057 \\
\hline SFA & $596.66 \pm 55.95$ & $455.86 \pm 39.07$ & 0.066 \\
\hline UFA & $1,164.40 \pm 93.97$ & $899.94 \pm 78.20$ & 0.053 \\
\hline MUFA & $740.28 \pm 78.29$ & $544.33 \pm 62.48$ & 0.078 \\
\hline PUFA & $424.11 \pm 18.34$ & $355.61 \pm 16.73$ & $0.017^{\star}$ \\
\hline n3 PUFA & $61.79 \pm 2.09$ & $53.26 \pm 0.95$ & $0.004^{\star \star}$ \\
\hline n6 PUFA & $362.32 \pm 16.98$ & $302.35 \pm 15.90$ & $0.024^{*}$ \\
\hline n6/n3 & $5.87 \pm 0.21$ & $5.65 \pm 0.18$ & 0.448 \\
\hline PUFA/MUFA & $0.60 \pm 0.04$ & $0.67 \pm 0.04$ & 0.240 \\
\hline PUFA/UFA & $0.37 \pm 0.02$ & $0.40 \pm 0.02$ & 0.255 \\
\hline PUFA/SFA & $0.74 \pm 0.04$ & $0.79 \pm 0.03$ & 0.293 \\
\hline MUFA/UFA & $0.63 \pm 0.02$ & $0.60 \pm 0.02$ & 0.255 \\
\hline SFA\% & $0.34 \pm 0.00$ & $0.34 \pm 0.00$ & 0.814 \\
\hline UFA\% & $0.66 \pm 0.00$ & $0.66 \pm 0.00$ & 0.815 \\
\hline MUFA\% & $0.42 \pm 0.01$ & $0.40 \pm 0.01$ & 0.255 \\
\hline PUFA\% & $0.25 \pm 0.01$ & $0.27 \pm 0.01$ & 0.196 \\
\hline
\end{tabular}

Data are shown as mean \pm SEM, ${ }^{*} p<0.05,{ }^{* *} p<0.01$.

ToFA, total fatty acids; SFA, saturated fatty acids; UFA, unsaturated fatty acids; MUFA, monounsaturated fatty acids; PUFA, ployunsaturated fatty acids; PUFA/MUFA, PUFA-toMUFA ratio; PUFAUIFA, PUFA-to-UFA ratio; MUFAUIFA, MUFA-to-UFA ratio.

\section{DISCUSSION}

At present, healthy diet and exercise are recognized as the most safe, effective and economical way to prevent obesity, but the issue that needs to be solved urgently is the increase of appetite $(18,19)$. Studies have shown hypoxia itself can cause a decrease in appetite and energy intake (20). Till date, epidemiological studies using large scale databases show a negative relationship between habitation in hypoxic conditions and obesity, without clear underlying mechanisms. Recently, it has been reported that $\mathrm{O}_{2}$ variations in organic systems may lead to considerable (3\%) weight loss and improve metabolic and cardiorespiratory health (21-23). This suggests that sustained hypoxia may benefit obese individuls' weight management $(24,25)$. In the present study, we have found that hypoxic training has decreased body weight and fat mass of HFD-induced obese mice and has improved glucose tolerance and fatty liver. We have also shown that metabolites related to glucose and lipid metabolism in the liver have been changed after hypoxic training.
In recent studies, intermittent hypoxia has been used as an adjuvant therapy to enhance weight loss in obese patients; additional weight loss was achieved when combined with exercise $(26,27)$. Human studies have shown that hypobaric hypoxic living conditions have decreased body weight in obese subjects (4). Hypoxia might lead to negative energy balance with weight loss through reducing energy intake and increasing energy expenditure (22). But the mechanism is still to be further studied. One small trial in humans indicate that low intensity exercise training in normobaric hypoxia may lead to more weight loss than normoxic training (21). Furthermore, studies have shown that several weeks of moderate exercise in normoxic conditions resulted in more body weight and fat mass reductions in obese persons compared to the same relative intensity in normobaric hypoxic conditions $(23,28)$. Animal studies shown that normobaric hypoxia plus exercise training resulted in lower adiposity in HFD Sprague-Dawley rats, and altered the adipose tissue leptin/leptin receptor (24). Our data demonstrate that hypoxic training can decrease body weight and fat mass in mice, which is consistent with the results of previous studies, and suggests that hypoxic training decreases body weight by fat mass loss.

Previous studies have shown that both exercise training and prolonged intermittent normobaric hypoxia have improved glucose tolerance in male Sprague-Dawley rats, and the improvement by hypoxia treatment was significantly greater than that with exercise training alone. (29). Groote et al., found that training in hypobaric hypoxia improved normoxic glucose tolerance in adolescents with obesity (30). In this study, after 4 weeks of hypoxic training, we observed improved glucose tolerance and insulin resistance in HFD-induced obese mice. The improvement of glucose tolerance and insulin resistance by hypoxic training seems to be partly related to the reduction in body weight.

Animal studies have shown that moderate exercise can decrease serum ALT activity, reduce liver injury and fibrosis by inhibiting macrophage infiltration, prevent liver steatosis and effectively improve the pathogenesis of non-alcoholic fatty liver disease in DIO mice (31). In addition, hypobaric hypoxia and intermittent normobaric hypoxia can reduce not only body weight by increasing leptin concentration, but also decrease blood cholesterol, and prevent liver steatosis in obese mice (26). Our current study observed the combined effects of normobaric hypoxic conditions and exercise training on the liver in HFDinduced obese mice. We found that both hypoxia and hypoxic training significantly reduced ALT activity, which suggested that liver injury was alleviated after intervention. Furthermore, liver morphology staining results showed a significant reduction in lipid droplets in hepatocytes in the hypoxic training group. This suggests that hypoxic training can reduce liver lipid accumulation and ameliorate fatty liver in HFD-induced obese mice. These all illustrated that although both hypoxia and normoxic training can partly improve fatty liver, hypoxic training intervenes with the optimal effect.

To further explore how hypoxic training specifically affects liver glucose and lipid metabolism, the current study has 

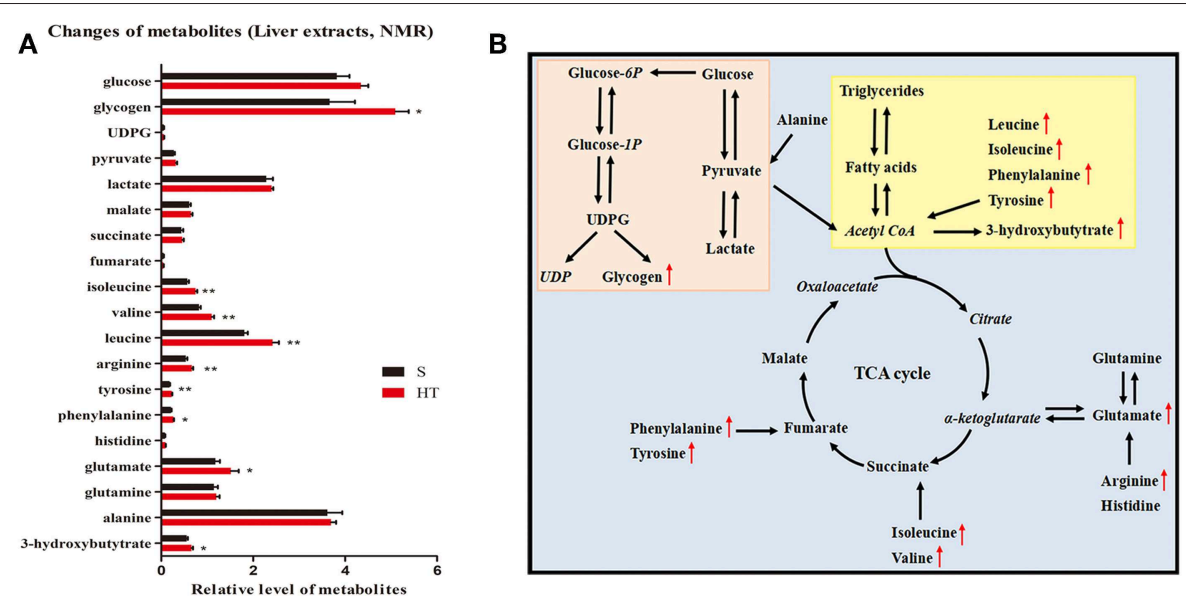

FIGURE 5 | Effects of hypoxic training on glucose and lipid metabolism. Changes of metabolites in response to hypoxic training in mice (A). Data are shown as mean $\pm \mathrm{SEM}, n=8,{ }^{*} p<0.05,{ }^{* *} p<0.01$. (B) Summary of metabolic pathways associated with hypoxia training intervention. The italic metabolite represents the undetected metabolite, and the red font is the significantly increased metabolite of Group D vs. Group A. The pink box is glucose metabolism pathway, and the yellow box is lipid metabolism pathway.

applied metabolomics. Our data demonstrated that hypoxic training decreased the levels of saturated fatty acid C18, n3 PUFA and n6 PUFA in liver, and increased the level of 3hydroxybutytrate. These results suggest that hypoxic training regulates fatty acid metabolism in the liver of HFD-induced obese mice. Studies have shown that branched-chain amino acids could increase glycogen synthesis in liver $(32,33)$. NMR results indicate that hypoxic training increases the levels of glycogen and branched-chain amino acids in the liver of HFDinduced obese mice. Taken together, our data reveals that hypoxic training promotes liver glucose metabolism in HFD-induced obese mice.

In conclusion, hypoxic training of 4 weeks has reduced body weight, improved glucose tolerance, rescued fatty liver and changed the glucose and lipid metabolites in HFD-induced obese mice. Future studies with exposure to hypoxia are warranted to compare the effects of various exercise modalities/intensities on changes in gait pattern. The transgenic models under or overexpressing genes involved in the oxygen transport system and crucial metabolic pathways can be of valuable interest for future research in this domain.

\section{DATA AVAILABILITY}

The raw data supporting the conclusions of this manuscript will be made available by the authors, without undue reservation, to any qualified researcher.

\section{ETHICS STATEMENT}

This study was carried out in accordance with the recommendations of Science Research Ethics Committee at the Shanghai University of Sports (No. 2015013). The protocol was approved by the Animal Care and Use Committee at the Shanghai University of Sports.

\section{AUTHOR CONTRIBUTIONS}

SG helped conceive the design, performed the NMR and GCFID-MS analyses, analyzed the data, and wrote the first draft of the manuscript. HT and YH performed other data analyses and helped to draft the manuscript. QY helped conceive the design and supervised experimental trials and training sessions. $\mathrm{KZ}$ and $\mathrm{C}-\mathrm{HK}$ assisted with data analyses and helped draft the manuscript. $\mathrm{SH}$ interpreted the study results and edited the manuscript. RW, PC, and TL helped conceive the design, assisted with data analyses, provided funding for the study, and helped draft the manuscript. All authors have read and approved the final version of the manuscript and agree with the order of presentation of the authors.

\section{FUNDING}

This research was supported by the National Natural Science Foundation of China $(81472148,31671242)$, the National Key R\&D Program of China (No. 2018YFC1314701), the Open Research Fund of the National Key Laboratory of Genetic Engineering (SKLGE1805), the Major Research Plan of the National Natural Science Foundation of China (91749104), the Emergency Management Project of the National Natural Science Foundation of China (31842034), the Shanghai Pujiang Talent Project (18PJ1400700), the Science and Technology Innovation Action Plan of Shanghai Science and Technology Committee (18140901300), the Open Research Fund of the National Key Laboratory of Genetic Engineering (SKLGE1803), and the Open Research Fund of the State Key Laboratory of Pharmaceutical Biotechnology (KF-GN-201701). 


\section{REFERENCES}

1. Trayhurn P. Hypoxia and adipose tissue function and dysfunction in obesity. Physiol Rev. (2013) 93:1-21. doi: 10.1152/physrev.00017.2012

2. Quintero P, Gonzalez-Muniesa P, Garcia-Diaz DF, Martinez JA. Effects of hyperoxia exposure on metabolic markers and gene expression in 3T3-L1 adipocytes. J Physiol Biochem. (2012) 68:663-9. doi: 10.1007/s13105-012-0169-8

3. Gonzalez-Muniesa P, Garcia-Gerique L, Quintero P, Arriaza S, LopezPascual A, Martinez JA. Effects of hyperoxia on oxygen-related inflammation with a focus on obesity. Oxid Med Cell Longev. (2015) 2015:8957827. doi: $10.1155 / 2016 / 8957827$

4. Lippl FJ, Neubauer S, Schipfer S, Lichter N, Tufman A, Otto B, et al. Hypobaric hypoxia causes body weight reduction in obese subjects. Obesity. (2012) 18:675-81. doi: 10.1038/oby.2009.509

5. Lopez-Pascual A, Bes-Rastrollo M, Sayón-Orea C, Perez-Cornago A, DíazGutiérrez J, Pons JJ, et al. Living at a geographically higher elevation is associated with lower risk of metabolic syndrome: prospective analysis of the SUN Cohort. Front Physiol. (2016) 7:658. doi: 10.3389/fphys.2016.00658

6. Hobbins LG, Hunter S, Gaoua N, Girard O. Normobaric hypoxic conditioning to maximise weight-loss and ameliorate cardio-metabolic health in obese populations: a systematic review. Am J Physiol Regul Integr Comp Physiol. (2017) 313:R251-64. doi: 10.1152/ajpregu.00160.2017

7. Vogt $M$, Hoppeler $H$. Is hypoxia training good for muscles and exercise performance? Prog Cardiovasc Dis. (2010) 52:525-33. doi: 10.1016/j.pcad.2010.02.013

8. Wang JS, Chen LY, Fu LL, Chen ML, Wong MK. Effects of moderate and severe intermittent hypoxia on vascular endothelial function and haemodynamic control in sedentary men. Eur J Appl Physiol. (2007) 100:12735. doi: 10.1007/s00421-007-0409-8

9. Nagahisa H, Mukai K, Ohmura H, Takahashi T, Miyata H. Effect of highintensity training in normobaric hypoxia on thoroughbred skeletal muscle. Oxid Med Cell Longev. (2016) 2016:1535367. doi: 10.1155/2016/1535367

10. Favret F, Henderson KK, Richalet JP, Gonzalez NC. Effects of exercise training on acclimatization to hypoxia: systemic $\mathrm{O}_{2}$ transport during maximal exercise. J Appl Physiol (1985). (2003) 95:1531-41. doi: 10.1152/japplphysiol.01220.2001

11. Di Meo S, Iossa S, Venditti P. Improvement of obesity-linked skeletal muscle insulin resistance by strength and endurance training. J Endocrinol. (2017) 234:R159-81. doi: 10.1530/JOE-17-0186

12. Roh HT, So WY. The effects of aerobic exercise training on oxidantantioxidant balance, neurotrophic factor levels, and blood-brain barrier function in obese and non-obese men. J Sport Health Sci. (2017) 6:447-53. doi: 10.1016/j.jshs.2016.07.006

13. Uddin GM, Youngson NA, Sinclair DA, Morris MJ. Head to head comparison of short-term treatment with the $\mathrm{NAD}(+)$ precursor Nicotinamide Mononucleotide (NMN) and 6 weeks of exercise in obese female mice. Front Pharmacol. (2016) 7:258. doi: 10.3389/fphar.2016.00258

14. Jiang L, Huang J, Wang Y, Tang H. Metabonomic analysis reveals the CCl4induced systems alterations for multiple rat organs. Phys Rev A. (2012) 11:1354-62. doi: 10.1021/pr3003529

15. Duan Y, An Y, Li N, Liu B, Wang Y, Tang H. Multiple univariate data analysis reveals the inulin effects on the high-fat-diet induced metabolic alterations in rat myocardium and testicles in the preobesity state. J Proteome Res. (2013) 12:3480. doi: $10.1021 / \mathrm{pr} 400341 \mathrm{f}$

16. Li H, An Y, Zhang L, Lei H, Zhang L, Wang Y, et al. Combined NMR and GC-MS analyses revealed dynamic metabolic changes associated with the carrageenan-induced rat pleurisy. J Proteome Res. (2013) 12:5520-34. doi: $10.1021 / \mathrm{pr} 400440 \mathrm{~d}$

17. Li X, Fang P, Mai J, Choi ET, Wang H, Yang XF. Targeting mitochondrial reactive oxygen species as novel therapy for inflammatory diseases and cancers. J Hematol Oncol. (2013) 6:19. doi: 10.1186/1756-8722-6-19

18. Castro EA, Peinado ABL, Cupeiro, Benito PJ, Szendrei, Butragueño, et al. What is the most effective exercise protocol to improve cardiovascular fitness in overweight and obese men? J Sport Health Sci. (2016) 8:25. doi: 10.1016/j.ramd.2014.10.014
19. Wiklund P. The role of physical activity and exercise in obesity and weight management: time for critical appraisal. J Sport Health Sci. (2016) 5:151-4. doi: 10.1016/j.jshs.2016.04.001

20. Mortola JP. Implications of hypoxic hypometabolism during mammalian ontogenesis. Respir Physiol Neurobiol. (2004) 141:345-56. doi: 10.1016/j.resp.2004.01.011

21. Netzer NC, Chytra R, Küpper T. Low intense physical exercise in normobaric hypoxia leads to more weight loss in obese people than low intense physical exercise in normobaric sham hypoxia. Sleep Breath. (2008) 12:129-34. doi: 10.1007/s11325-007-0149-3

22. Kayser B, Verges S. Hypoxia, energy balance and obesity: from pathophysiological mechanisms to new treatment strategies. Obes Rev. (2013) 14:579-92. doi: 10.1111/obr.12034

23. Kong Z, Zang Y, Hu Y. Normobaric hypoxia training causes more weight loss than normoxia training after a 4-week residential camp for obese young adults. Sleep Breath. (2014) 18:591-7. doi: 10.1007/s11325-0130922-4

24. Lu Y, Feng L, Xie M, Zhang L, Xu J, He Z, et al. Hypoxic living and exercise training alter adipose tissue leptin/leptin receptor in rats. Front Physiol. (2016) 7:554. doi: 10.3389/fphys.2016.00554

25. Millet GP, Debevec T, Brocherie F, Malatesta D, Girard O. Therapeutic use of exercising in hypoxia: promises and limitations. Front Physiol. (2016) 7:224. doi: 10.3389 /fphys.2016.00224

26. Urdampilleta A, Gonzalez-Muniesa P, Portillo MP, Martinez JA. Usefulness of combining intermittent hypoxia and physical exercise in the treatment of obesity. J Physiol Biochem. (2012) 68:289-304. doi: 10.1007/s13105-011-0115-1

27. Verges S, Chacaroun S, Godin-Ribuot D, Baillieul S. Hypoxic conditioning as a new therapeutic modality. Front Pediatr. (2015) 3:58. doi: $10.3389 /$ fped.2015.00058

28. Wiesner S, Haufe S, Engeli S, Mutschler H, Haas U, Luft FC, et al. Influences of normobaric hypoxia training on physical fitness and metabolic risk markers in overweight to obese subjects. Obesity (Silver Spring). (2010) 18:116-20. doi: 10.1038/oby.2009.193

29. Chiu LL, Chou SW, Cho YM, Ho HY, Ivy JL, Hunt D, et al. Effect of prolonged intermittent hypoxia and exercise training on glucose tolerance and muscle GLUT4 protein expression in rats. J Biomed Sci. (2004) 11:838-46. doi: 10.1007/BF02254369

30. De Groote E, Britto FA, Bullock L, Francois M, Buck CDE, Nielens $\mathrm{H}$, et al. Hypoxic training improves normoxic glucose tolerance in adolescents with obesity. Med Sci Sports Exerc. (2018) 50:2200-8. doi: 10.1249/MSS.0000000000001694

31. Kawanishi N, Yano H, Mizokami T, Takahashi M, Oyanagi E, Suzuki K. Exercise training attenuates hepatic inflammation, fibrosis and macrophage infiltration during diet induced-obesity in mice. Brain Behav Immun. (2012) 26:931-41. doi: 10.1016/j.bbi.2012. 04.006

32. Zhang Y, Guo K, LeBlanc RE, Loh D, Schwartz GJ, Yu YH. Increasing dietary leucine intake reduces diet-induced obesity and improves glucose and cholesterol metabolism in mice via multimechanisms. Diabetes. (2007) 56:1647-54. doi: 10.2337/db07-0123

33. Tajiri K, Shimizu Y. Branched-chain amino acids in liver diseases. World J Gastroenterol. (2013) 19:7620-9. doi: 10.3748/wjg.v19.i 43.7620

Conflict of Interest Statement: The authors declare that the research was conducted in the absence of any commercial or financial relationships that could be construed as a potential conflict of interest.

Copyright $\odot 2019$ Wang, Guo, Tian, Huang, Yang, Zhao, Kuo, Hong, Chen and Liu. This is an open-access article distributed under the terms of the Creative Commons Attribution License (CC BY). The use, distribution or reproduction in other forums is permitted, provided the original author(s) and the copyright owner(s) are credited and that the original publication in this journal is cited, in accordance with accepted academic practice. No use, distribution or reproduction is permitted which does not comply with these terms. 